\title{
Performance of STT-Vegas in Heterogeneous Wired and Wireless Networks
}

\author{
Hong Zhou \\ University of Southern Queensland, Australia \\ hzhou@usq.edu.au \\ Amoakoh Gyasi-Agyei \\ Central Queensland University, Australia \\ gyasi-agyei@cqu.edu.au \\ Zhongwei Zhang \\ University of Southern Queensland, Australia \\ zhongwei@usq.edu.au
}

\begin{abstract}
TCP Vegas is a proactive congestion control mechanism proposed to improve TCP performance by using Round Trip Time (RTT) as a main parameter to monitor traffic condition and avoid congestion. However, TCP Vegas does not perform well on bidirectional links with unbalanced traffic, and on wireless links. A simple Single-Trip Time (STT) based modification to TCP Vegas, namely STT-Vegas, was introduced in [1] to improve the performance of TCP Vegas. It has been demonstrated that STT-Vegas outperforms Vegas in various network scenarios in wireline networks. This paper examines the performance of STT-Vegas in heterogeneous wired and wireless networks and investigates its possible enhancements in such networks.
\end{abstract}

Index Terms - Vegas, congestion control, STT, bi-direction unbalanced traffic.

\section{INTRODUCTION}

$\mathrm{T}$ HERE are two main transport layer protocols in an IP network, TCP (Transmission Control Protocol) and UDP (User Datagram Protocol). Totally different from simple connectionless UDP [2], TCP is a connectionoriented protocol specifically designed to support end-to-end reliable delivery of data [3]. Thus it is adopted by all Internet applications that require reliable transmission (e.g. TELNET, FTP, HTTP, and Email).

TCP was first formalized in RFC793 [4] two decades ago and several modern versions of TCP (e.g. Tahoe [5], Reno, New-Reno [6] etc.) have been proposed and implemented consequently. To achieve reliability, TCP provides algorithms including window-based congestion control and packet retransmission. As the most popular TCP currently in use, Reno TCP implements slow-start, congestion avoidance, fast retransmission and fast recovery functions. Though different from each other in fast retransmission algorithm, congestion control of these TCP versions is commonly based on additive increase multiplicative decrease (AIMD) [7]. That is, TCP increases the congestion window by roughly one segment per Round-Trip Time (RTT) when there is no packet loss detected and TCP reduces its window size by half for every window containing a packet loss.
TCP has worked reasonably well over the past several years. However, some weaknesses have been observed [1, 8 , 9]. TCP uses a reactive rather than proactive congestion control protocol. It cannot avoid congestion from happening. It constantly explores the maximum throughput until packet loss occurs, then retransmits the lost packet and reduces its transmission rate. It does not have any mechanism to detect the network condition except using packet loss as an implicit indication of network congestion. This also causes TCP to perform poorly in unreliable, high error-rate wireless networks. TCP treats all packet losses equally as it cannot distinguish the packet loss introduced due to congestion from link errors. TCP's weakness in congestion control has not attracted adequate attention until in recent years as wireless Internet applications have become more and more popular.

TCP Vegas [10] represents a positive step to deal with the congestion problem in wireline networks. Vegas tries to detect network traffic condition and avoid congestion and packet loss from happening. In Vegas's congestion avoidance mechanism, it monitors packets' RTT and uses the RTT to estimate the traffic condition in the network. Two thresholds, namely, $\alpha$ and $\beta$, are defined in TCP Vegas [10]. Thus it can classify the traffic condition into three categories. Intuitively, we call them as light, moderate and congested traffic conditions. Different from TCP Reno's AIMD, Vegas increases the congestion window linearly during the next RTT when the extra queued data is less than the lower threshold $\alpha$; Vegas decreases the congestion window linearly when the extra queued data is greater than the higher threshold; otherwise Vegas leaves the window unchanged. Along with Vegas's new retransmission and slow-start mechanisms, Vegas can not only achieve higher throughput but also reduce the packet loss significantly.

However, similar to other TCP versions (e.g. Reno), Vegas does not solve the problem of performance degradation in traffic asymmetric and wireless networks. We observed that Vegas cannot use the available bandwidth efficiently in the situation when the traffic in the forward direction (i.e. from a source to its destination) and the traffic in the backward direction (i.e. from the destination to its source) are seriously unbalanced. This situation could be encountered often in asymmetric networks. By using 
conventional RTT only, Vegas cannot distinguish the congestion in the forward direction from that in the backward direction. In the case of congestion in the backward direction only, Vegas unnecessarily reduces its congestion window and thus suffers from very low throughput.

A simple Single-Trip Time (STT) based modification to improve Vegas in such a network, namely STT-Vegas, was proposed in [1]. It was shown that, by more closely monitor the traffic condition, STT-Vegas can achieve more efficient utilization of bandwidth and outperform Vegas not only in a network with unbalanced traffic in bi-directions but also in other normal network scenarios. This paper examines the performance of STT-Vegas and possible enhancements in heterogeneous wired and wireless networks.

Several schemes have been proposed to address congestion control problem in wireless networks $[3,4]$. Examples include Explicit Loss Notification (ELN) [5], Explicit Congestion Notification (ECN), Indirect TCP [7] and Snoop [8]. In general, these proposals to overcome the problem are based on two fundamental ideas: (i) decoupling the congestion control from retransmission of lost packets and (ii) retransmission of the lost packets as early/closely to the wireless link as possible. ELN and ECN can provide the TCP sender with the reasons for packet loss. Indirect TCP decomposes the TCP connection into two sub-connections for the wired and wireless parts of the path. The Snoop protocol, a so-called link layer proposal, introduces a snoop agent at the base station. The agent monitors every packet that passes in both directions and maintains a cache of TCP segments that have not yet been acknowledged by the receiver. It detects packet loss by duplicate ACKs or a local Retransmission TimeOut (RTO), and retransmits the lost packet. It has been shown that the Snoop protocol is an effective mechanism to improve the throughput performance of TCP in a wireless network.

In the simulation studies, we combined STT-Vegas with the Snoop protocol [8]. Simulation results show that the combined mechanism performs well in various network scenarios comparing with Vegas and Reno. In particular, it is shown that STT-Vegas can substantially improve the throughput of a highly traffic asymmetric network.

The rest of this paper is organized as follows. Section II briefly describes the congestion avoidance mechanism of TCP Vegas, STT-Vegas and the Snoop. Section III introduces the simulation model and presents the simulation results showing the dynamic behaviour of TCP versions in four different network scenarios. Section IV summarizes the results and concludes the paper.

\section{TCP VEGAS AND STT-VEGAS}

\section{A. TCP Vegas}

TCP Vegas is a modification of TCP Reno introduced by Brakmo and Peterson in 1994 [10]. Vegas employs three techniques to increase throughput and decrease the packet loss, a new retransmission mechanism, a congestion avoidance mechanism and a modified slow-start mechanism. As only the congestion avoidance mechanism is relevant to the issue in an asymmetric network, we only briefly summarize the congestion avoidance mechanism in this section.

Vegas's approach is to measure and control the amount of extra packets, namely queued packets, the connection has in transit. It tries to maintain the queued packets at a targeted range (between thresholds $\alpha$ and $\beta$ ). The details of the mechanism are described below. For every round trip time,

1. Calculate the expected throughput. Expected throughput is defined by

$$
\text { Expected }=\text { WindowSize / BaseRTT, }
$$

where BaseRTT is defined as the minimum of all measured RTTs.

2. Calculate the actual sending rate, which is defined by Actual $=$ Segments in Transit / RTT,

where Segments in Transit is the number of segments that are transmitted during which a segment is sent and its acknowledgement is received.

3. Compare the actual to expected, and adjust the window accordingly. Let

$$
\text { Diff = expected } \text {-actual }
$$

If Diff $<\alpha$, Vegas increases the congestion window linearly during the next RTT; if Diff $<\beta$, Vegas decreases the congestion window linearly; if $\alpha<$ Diff $<\beta$ Vegas leaves the window unchanged.

\section{B. STT-Vegas}

STT-Vegas [1] borrows the ideas and design goals in TCP Vegas. However, STT-Vegas introduced STT in the calculation of RTTs. STT is defined as the time difference between when a packet is generated at the source and when it is received at destination. Simple modifications to Vegas are needed at both the source and destination ends. For every round trip time, the F-STTs (i.e. STTs in the forward direction) are recorded at the destination and sent back to the source end in the ACK headers. We define

$$
\text { BaseRTT }=\text { F-BaseSTT }+ \text { B-BaseSTT }
$$

where $F$-BaseSTT and B-BaseSTT represents minimum single-trip time in forward and backward directions respectively. The B-BaseSTT is calculated based on the available F-BaseSTT and BaseRTT. To eliminate the unnecessary impact of congestion in the backwards direction, it is defined that

$$
R T T^{\prime}=F-S T T+B-\text { BaseSTT }
$$

Then, by using $R T T^{\prime}$ obtained in Equation (5), the values of the actual and the expected are calculated as stated in Equations (1) and (2). Finally, STT-Vegas compares the values of the actual and the expected and adjusts the congestion window accordingly. Note that, although singletrip time (i.e. F-STT) is required, we only use the sum $R T T^{\prime}$ in Equation (5) to calculate the actual and expected queued packets and thus the clocks at the source and destination ends are not necessarily synchronized.

\section{NETWORK SCENARIO AND SIMULATION MODEL}

The network scenario is shown in Figure 1. We assume that the fixed host $(\mathrm{FH})$ sends TCP data to the mobile hosts (MH). Without loss of generality, suppose the TCP packets traverse a ingress edge router (IER), a core router (CR) and a base station (BS) from $\mathrm{FH}$ to the $\mathrm{MH}$. The $\mathrm{MH}$ is assumed to be located in a wireless LAN. In this paper, mobility 
issues are not considered. We focus on the impacts of link loss on the performance of TCP Reno, Vegas and STTVegas. Hence, a lossy link is used to represent wireless environment.

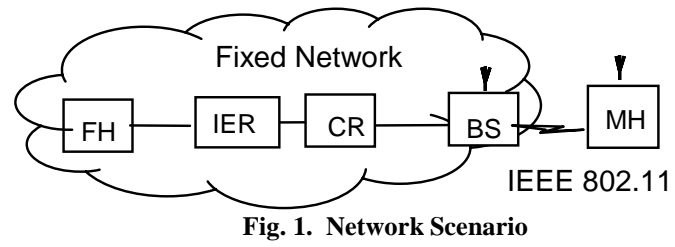

The simulation topology is shown in Fig. 2. UDP traffic is used here as background traffic to assist in investigating whether STT-Vegas can monitor and control the network condition. To investigate the performance of STT-Vegas under different network conditions, four network scenarios are chosen. They are: 1). TCP traffic only, without any forward and backward UDP traffic. This represents a symmetric network. 2). With forward UDP traffic but without any backward UDP traffic. This represents an asymmetric network with traffic congestion in the forward direction. 3).Without forward UDP traffic but with backward UDP traffic. This represents an asymmetric network with traffic congestion in the backward direction. This is the particular case in which Vegas performs poorly and STT-Vegas is targeted to improve. 4).With both forward and backward UDP traffic. In the rest of this paper, we use F0B0 to represent the first network scenario and similarly use F1B0, F0B1, and F1B1 for the other three network scenarios.

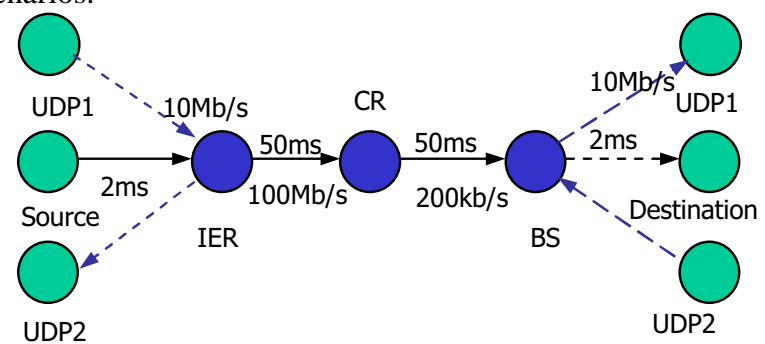

Fig. 2. Simulation Model

We implemented STT-Vegas based on the Vegas implementation in ns2. The bandwidths and propagation delays are given in Fig. 2. Other relevant parameters are given in Table 1.

\section{Simulation Results}

We ran the simulations for each TCP variation (Reno, Vegas, and STT-Vegas) with and without the Snoop at four different network scenarios addressed in the previous section. This section presents some typical simulation results on sequence number, throughput, congestion window, queue length under different TCP schemes.

In Section $\mathrm{A}$ and $\mathrm{B}$, the results with and without the Snoop obtained in the same scenario F1B1 are presented respectively. In Scenario F0B0, TCP-Vegas and STT-Vegas have very similar performance and both perform better than TCP Reno in general. In Section $\mathrm{C}$, the results in the scenario F0B1, a special case, are presented. In each section, the performances of each TCP version are compared.

\section{A. Without the Snoop}

The simulation results of goodput for three TCP versions without the Snoop are presented in Fig. 3 respectively. The goodput is the number of bits of TCP packets which are transmitted at the source and successfully received at the destination divided by the duration of the transmission.

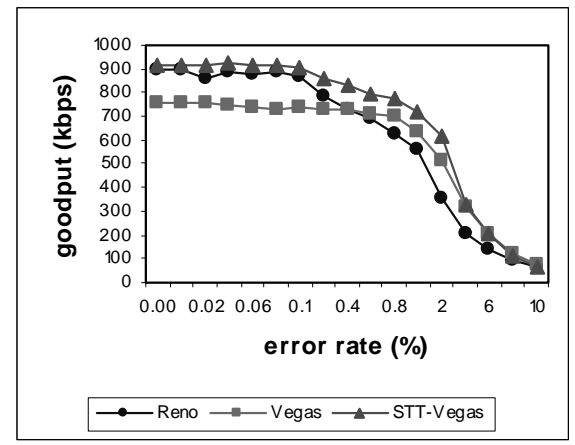

Fig. 3. Comparison of three TCP implementations

As shown in Fig. 3, STT-Vegas has better performance than Vegas with lower error rates $(0.01 \%-2 \%)$. When the error rates are high (4\%-10\% link loss rates), three TCP implementations have similar performance. The goodput of two Vegas versions drops slower than Reno when the link loss rate increases. This is because Vegas adjust window size based on the estimated traffic condition rather than packet loss. However, as the error rate increases, the significant number of packet losses contributes to inaccurate estimation of the traffic condition (i.e. inaccurate RTTs).

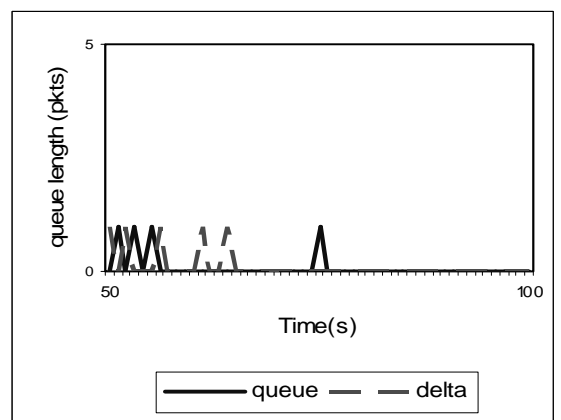

(a) STT-Vegas

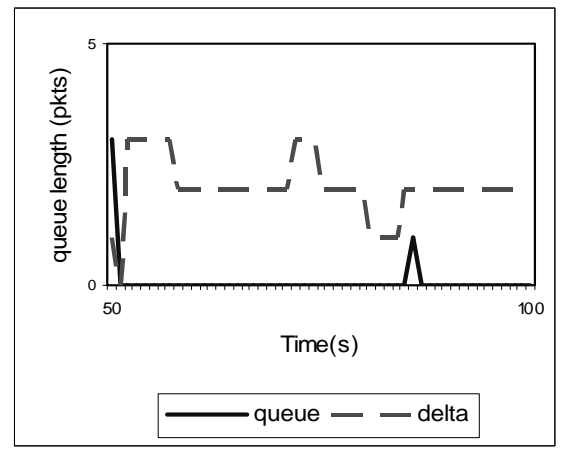

(b) Vegas

Fig. 4. Comparison of queue length and estimated traffic condition

Fig. 4 (a) and (b) give the queue length of the egress edge router (Qиеие) and estimated traffic condition (delta) for Vegas and STT-Vegas at link error rate of $1 \%$, respectively. Delta in Fig. 4 represents the value of Diff defined in Section II. Comparing Fig. 4 (a) and (b), we can observe that STT- 
Vegas can track the traffic condition (Queue) more closely than Vegas. This is the main reason that STT-Vegas can improve Vegas's throughput.

\section{B. With the Snoop}

The simulation results for three TCP versions with the Snoop protocol are presented in Fig. 5.

Comparing Fig. 3 and Fig 5, the Snoop can improve Reno's performance when the link error rate is higher than $0.1 \%$, while the Snoop has noticeable improvement for two Vegas implementations when the link error rate is higher than $1 \%$. When the link error rate is higher than $1 \%$ Reno TCP has better performance than Vegas and STT-Vegas although the packet loss rate no. of Reno is higher than those of Vegas and STT-Vegas. From Fig. 5, we can observe that STT-Vegas performs better than Vegas when the link error rate is lower than $2 \%$. With higher link error rates, STTVegas and Vegas have similar performance.

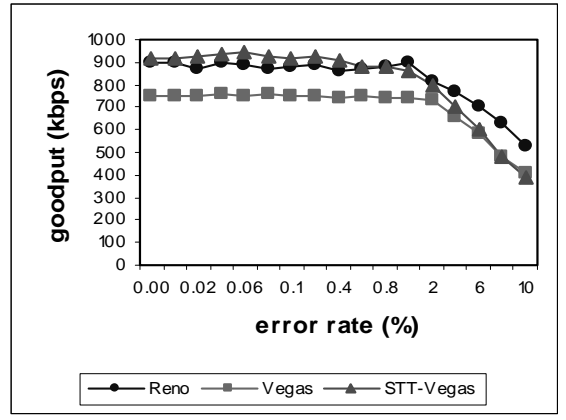

Fig. 5. Comparison of three TCP implementations

\section{A Special Case (FOB1)}

This section presents simulation results for the network scenario F0B1, i.e., there is no congestion in the forward data direction while in the backward direction, traffic congestion occurs. In this network scenario, UDP1 source sends exponentially distributed traffic to its destination. In this case, Vegas gives wrong traffic condition estimation based on only RTTs. Vegas misinterprets there is a congestion in the forward direction thus it suffers very low goodput regardless link error rate and the use of the Snoop protocol. In the case without the Snoop, STT-Vegas has slightly better performance than Reno at the link error rates between $0.4 \%-4 \%$. In the case with the Snoop, STT-Vegas has similar performance with Reno until the link error rate reaches above $1 \%$. Again this shows that combining Vegas implementations with the Snoop is not as efficient as combining Reno with the Snoop.

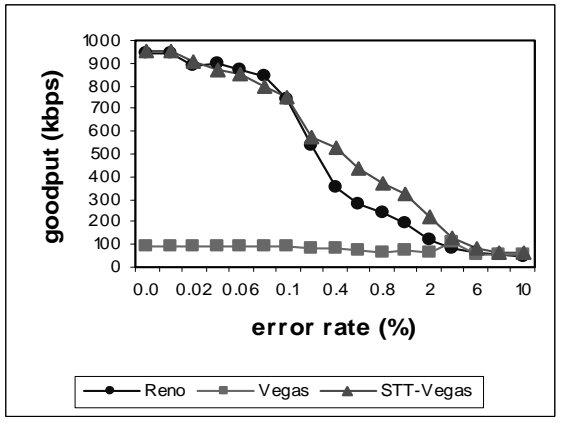

(a) without the Snoop

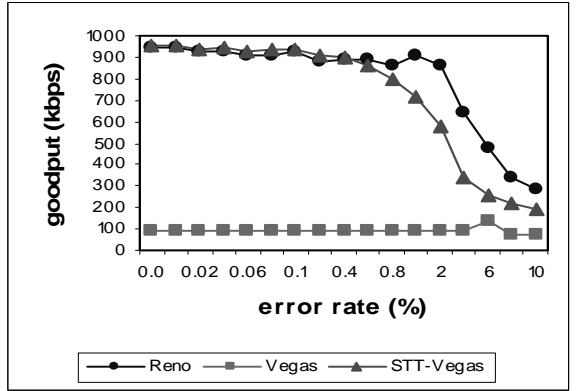

(b) with the Snoop

Fig. 6. Comparison of goodput in scenario F0B1

\section{SUMMARY AND FUTURE WORK}

This paper compares the performance of three TCP implementations in heterogeneous wired and wireless networks. The simulation results show that STT-Vegas performs better than Vegas in various network scenarios and STT-Vegas performs better than Reno with lower link error rates in general. Combining Vegas implementations with the Snoop will improve the performance significantly at high packet error rates $(>2 \%)$. However, with error rates less than $2 \%$, no significant differences are observed comparing with and without the Snoop. Simply combining Vegas implementations with the Snoop is not as efficient as combining Reno with the Snoop. An enhancement which uses the variations of STTs to distinguish the link and congestion losses can be easily integrated in STT-Vegas. Its performance will be analyzed in the future studies.

\section{REFERENCES}

[1] H. Zhou, "STT-Vegas: A Simple Single-Trip Time Based Modification of Vegas," presented at IEEE Consumer Communications and Networking Conference 2006 Las Vegas, Nevada, USA, 2006.

[2] J. Postel, "User Datagram Protocol," Internet Engineering Task Force (IETF) RFC 768, 1980.

[3] A. S. Tanenbaum, Computer Networks, Third ed: Prentice-Hall International, 1996.

[4] I. S. I. University of Southern California, "Transmission Control Protocol," Internet Engineering Task Force (IETF) RFC 793, 1981.

[5] V. Jacobson, "Congestion Avoidance and Control," presented at ACM SIGCOMM, 1988.

[6] S. Floyd, "TCP and Explicit Congestion Notification," ACM Comput. and Commun. Rev., vol. 24, pp. 10-23, 1994.

[7] W. R. Stevens, TCP/IP Illustrated, Volumn 1 (The Protocols): Addison-Wesley, 1994.

[8] H. Balakrishnan, V. N. Padmanabhan, S. Seshan, and R. H. Kaze, "A Comparison of Improving TCP Performance over Wireless Links," IEEE/ACM Trans. Networking, vol. 5, pp. 756-769, 1997.

[9] S. Ryu, C. Runp, and C. Qiao, "Advances in Internet Congestion Control," IEEE commun. Surveys \& Turorials, vol. 5, pp. 28-39, 2003.

[10] L. S. Brakmo and L. L. Peterson, "TCP Vegas: End to End Congestion Avoidance on A Global Internet," IEEE J. on Selected Areas in Commun., vol. 13, pp. 1465 - 1480, 1995. 